\title{
A NOTE ON THE FRACTIONAL CALCULUS
}

\author{
by DONALD I. CARTWRIGHT and JOHN R. MCMULLEN \\ (Received 2nd November 1976)
} 379).

We prove the following theorem, which settles a conjecture made by J. S. Lew (2, p.

Theorem. Let $E$ be the space $L^{p}[0,1](1 \leqslant p<\infty)$, or $C[0,1]$. Then there is precisely one family $\left(I_{\alpha}\right)_{\alpha>0}$ of operators on $E$ satisfying the following conditions:

(i) $\left(I_{1} f\right)(x)=\int_{0}^{x} f(t) d t$ for all $f \in E$ and $x \in[0,1]$,

(ii) $I_{\alpha} I_{\beta}=I_{\alpha+\beta}$ for all $\alpha, \beta>0$,

(iii) $I_{\alpha} \geqslant 0$ for all $\alpha>0$ (i.e. $f \in E$ and $f \geqslant 0 \Rightarrow I_{\alpha} f \geqslant 0$ ),

(iv) $\alpha \mapsto I_{\alpha}$ is a continuous map of $(0, \infty)$ into $\mathscr{L}(E)$ for some Hausdorff topology on $\mathscr{L}(E)$, weaker than the norm topology.

That family is given by the Riemann-Liouville formula

$$
\left(I_{\alpha} f\right)(x)=\frac{1}{\Gamma(\alpha)} \int_{0}^{x}(x-t)^{\alpha-1} f(t) d t
$$

Proof. The family of operators defined by (1) certainly satisfies these conditions, the continuity demanded in (iv) holding for the norm topology on $\mathscr{L}(E)$. The proof of the uniqueness is given over the complex field, since the result for real scalars may be deduced from the complex case.

The convolution of $f, g \in L^{\prime}[0,1]$ is given a.e. by

$$
(f * g)(x)=\int_{0}^{x} f(x-t) g(t) d t \quad(0 \leqslant x \leqslant 1) .
$$

Equipped with $*, L^{\prime}[0,1]$ becomes a commutative Banach algebra, and $E$ a Banach $L^{\prime}[0,1]$ module. A theorem of Titchmarsh [1, p. 39] shows that if $f_{1}, \ldots, f_{m} \in L^{\prime}[0,1]$, then $f_{1} * \cdots * f_{m}=0$ if and only if there are $\lambda_{k} \in[0,1](1 \leqslant k \leqslant m)$ such that $\sum_{k=1}^{m} \lambda_{k}=1$ and such that $f_{k}$ vanishes a.e. on $\left[0, \lambda_{k}\right]$.

For $g \in L^{1}[0,1]$ and $f \in E$, set $R_{g} f=g * f$. Then $R_{g} \in \mathscr{L}(E)$ and $\left\|R_{g}\right\| \leqslant\|g\|_{1}$. Titchmarsh's Theorem shows that $R_{g}$ is injective if $g$ vanishes a.e. on no interval $[0, \lambda]$ with $\lambda>0$, and that $g \mapsto R_{g}$ is an injective map of $L^{1}[0,1]$ into $\mathscr{L}(E)$. For $\alpha \in R, \alpha>0$, and for $x \in(0,1]$ define

$$
g_{\alpha}(x)=\frac{1}{\Gamma(\alpha)} x^{\alpha-1}
$$

and write $R_{\alpha}=R_{R_{a}}$. Thus $\left(R_{\alpha}\right)_{\alpha>0}$ is the family of operators defined by the right hand member of (1). 
Observe first that if $T \in \mathscr{L}(E)$ and if $T R_{1}=R_{1} T$, then for $k=1,2, \ldots$ we have $R_{1}^{k}=R_{k}$, and hence $T$ commutes with $R_{g}$ whenever $g$ is the restriction to $[0,1]$ of a polynomial. Density of such functions in $L^{1}[0,1]$ and continuity of $g \mapsto R_{g}$ entail that

$$
T R_{1}=R_{1} T \Rightarrow T R_{\mathrm{g}}=R_{\mathrm{g}} T \text { for all } g \in L^{1}[0,1] \text {. }
$$

We now show that if $T \in \mathscr{L}(E), m \in\{1,2,3, \ldots\}$ and $T^{m}=R_{1}$, then there is a complex $m$-th root of unity $\eta$ such that $T=\eta R_{1 / m}$. Suppose first that $T=R_{g}$ for some $g \in L^{1}[0,1]$. We have $T^{m}=R_{g}^{m}=R_{1}=R_{1 / m}^{m}$. Hence $0=R_{g}^{m}-R_{1 / m}^{m}=R_{h}$, where $h=$ $\left(g-\eta_{1} g_{1 / m}\right) * \cdots *\left(g-\eta_{m} g_{1 / m}\right)$ and $\eta_{1}, \ldots, \eta_{m}$ are the complex $m$-th roots of unity. Thus $h=0$ a.e. and hence either (i) $g$ agrees a.e. on $[0,1]$ with $\eta_{k} g_{1 / m}$ for some $k$ or else (ii) $g$ agrees a.e. with at least two $\eta_{k} g_{1 / m}$ on some interval $[0, \lambda], \lambda>0$. But $g_{1 / m}$ vanishes a.e. on no such interval, and so the first situation must obtain, as claimed.

Now we relax the hypothesis $T=R_{g}$. Since $T^{m}=R_{1}, T$ and $R_{1}$ commute, and hence $S=R_{1} T$ commutes with $R_{g}$ for all $g \in L^{1}[0,1]$. Put $g=T 1$. Then for all $f \in E$,

$$
S f=T R_{1} f=T R_{f} 1=R_{f} g=R_{\ell} f,
$$

so that $S=R_{g}$. Also $S^{m}=T^{m} R_{1}^{m}=R_{m+1}=\left(R_{1+(1 / m)}\right)^{m}$. The above argument shows that $g=\eta g_{1+(1 / m)}$ for some complex $m$-th root of unity $\eta$, and so

$$
R_{1} T=S=R_{g}=\eta R_{1} R_{1 / m}
$$

This implies that $T=\eta R_{1 / m}$, since $R_{1}$ is injective.

We have thus shown that the conditions $T^{m}=R_{1}$ and $T \geqslant 0$ are satisfied for a unique $T \in \mathscr{L}(E)$, namely $T=R_{1 / m}$. An obvious argument shows that if $\left(I_{\alpha}\right)_{\alpha>0} \subset \mathscr{L}(E)$ satisfies (i), (ii) and (iii), then $I_{\alpha}=R_{\alpha}$ for all positive rational $\alpha$, and condition (iv) implies this equality for all positive real $\alpha$, as required.

The theorem also holds when $E$ is the space of all locally $p$-th power integrable functions $(1 \leqslant p<\infty)$ [resp. $C^{k}$ functions $(0 \leqslant k \leqslant \infty)$, resp. distributions) on $\boldsymbol{R}$ whose supports are bounded on the left, provided the range of integration is $(-\infty, x)$ in (i) and (1). (In the distribution case these integrals are interpreted as the convolutions $g_{1} * f$ and $g_{\alpha} * f$ where $g_{\alpha}(x)$ is given by (2) for $x>0$ and set equal to zero for $x \leqslant 0$.) The proof in these cases is similar, except that instead of Titchmarsh's theorem, the fact is used that each of these spaces forms an integral domain with respect to convolution (3, p. 173).

\section{REFERENCES}

(1) I. C. GohBERG and M. G. KREINN, Theory and Applications of Volterra Operators in Hilbert Space (Translations of Mathematical Monographs, Vol. 24, American Mathematical Society, Providence, R.I., 1970).

(2) B. Ross (editor), Fractional Calculus and Its Applications, Proceedings of the International Conference Held at the University of New Haven, June 1974, (Lecture Notes in Mathematics, Vol. 457, Springer-Verlag, Berlin-New York, 1975).

(3) L. Schwartz, Théorie des Distributions (Hermann, Paris, 1966).

UNIVERSITY OF SYDNEY

SYDNEY, N.S.W. 2006

Australia 